\title{
BMJ Open Timing Of Primary Surgery for cleft palate (TOPS): protocol for a randomised trial of palate surgery at 6 months versus 12 months of age
}

\author{
William Shaw, ${ }^{1}$ Gunvor Semb, ${ }^{1}$ Anette Lohmander, ${ }^{2}$ Christina Persson, ${ }^{3}$ \\ Elisabeth Willadsen, ${ }^{4}$ Jill Clayton-Smith, ${ }^{5}$ Inge Kiemle Trindade, ${ }^{6}$ Kevin J Munro, ${ }^{7}$ \\ Carrol Gamble, ${ }^{8}$ Nicola Harman, ${ }^{8}$ Elizabeth J Conroy, ${ }^{8}$ Dieter Weichart, ${ }^{1}$ \\ Paula Williamson ${ }^{8}$
}

To cite: Shaw W, Semb G, Lohmander A, et al. Timing Of Primary Surgery for cleft palate (TOPS): protocol for a randomised trial of palate surgery at 6 months versus 12 months of age. BMJ Open 2019;9:e029780. doi:10.1136/ bmjopen-2019-029780

- Prepublication history and additional material for this paper are available online. To view these files, please visit the journal online (http://dx.doi. org/10.1136/bmjopen-2019029780).

Received 15 February 2019

Revised 16 May 2019

Accepted 17 May 2019
Check for updates

(C) Author(s) (or their employer(s)) 2019. Re-use permitted under CC BY-NC. No commercial re-use. See rights and permissions. Published by BMJ.

For numbered affiliations see end of article.

Correspondence to Professor William Shaw; bill.shaw@manchester.ac.uk

\section{ABSTRACT}

Introduction Cleft palate is among the most common birth abnormalities. The success of primary surgery in the early months of life is crucial for successful feeding, speech, hearing, dental development and facial growth. Over recent decades, age at palatal surgery in infancy has reduced. This has led to palatal closure in one-stage procedures being carried out around the age of 12 months, but in some cases as early as 6 months. The primary objective of the Timing Of Primary Surgery for Cleft Palate (TOPS)trial is to determine whether surgery for cleft palate performed at 6 or 12 months of age is most beneficial for speech outcomes.

Methods and analysis Infants with a diagnosis of non-syndromic isolated cleft palate will be randomised to receive standardised primary surgery (Sommerlad technique) for closure of the cleft at either 6 months or 12 months, corrected for gestational age. The primary outcome will be perceived insufficient velopharyngeal function at 5 years of age. Secondary outcomes measured across 12 months, 3 years and 5 years will include growth, safety of the procedure, dentofacial development, speech, hearing level and middle ear function. Video and audio recordings of speech will be collected in a standardised age-appropriate manner and analysed independently by multiple speech and language therapists. The trial aims to recruit and follow-up 300 participants per arm. Data will be analysed according to the intention-to-treat principle using a $5 \%$ significance level. All analyses will be prespecified within a full and detailed statistical analysis plan.

Ethics and dissemination Ethical approval has been sought in each participating country according to countryspecific procedures. Trial results will be presented at conferences, published in peer-reviewed journals and disseminated through relevant patient support groups. Trial registration number NCT00993551; Pre-results.

\section{INTRODUCTION}

Clefts of the lip and/or palate, occurring with an incidence of about 1 per 600 births, are among the most common birth anomalies.
Strengths and limitations of this study

- International trial covering speech development in children across Scandinavia, the UK and Brazil.

- Surgical repair was calibrated across surgeons who were all trained in the Sommerlad technique.

- Longitudinal speech assessments at 12 months, 3 years and 5 years will be independently analysed by multiple speech and language therapists whose ratings will be calibrated on practice recordings.

- Standardised assessments of additional outcomes include postoperative complications, hearing levels, middle ear function and dentofacial development.

- The study excludes coexisting conditions such as syndromic cleft palate or severe developmental delays that are known to adversely affect speech development or its assessment.

This trial will focus on isolated clefts of the palate, which occur with a global incidence of 4.5 per 10000 births. ${ }^{1}$ Depending on geographic location, the prevalence of isolated clefts of the palate ranges from 1.8 to 14.6 per $10000 .^{1}$

The timing of palatal surgery has been a controversial issue since the $1930 \mathrm{~s}^{2}{ }^{2}$ Traditionally, rationale for delaying hard palate surgery was partly based on the belief that postponing the trauma of palatal closure may reduce maxillary growth disturbance. However, there is little evidence that facial skeletal growth in individuals with isolated cleft palate is substantially affected by different surgical protocols, though maxillary arch form, especially transversely, may be affected. ${ }^{3-6}$

Over recent decades, the age at which palatal surgery is carried out has reduced. This has led to one-stage palatal closure within 12 months of age at cleft units in Europe and the USA. Protagonists of early closure of the 
palatal cleft have proposed that since speech is a learnt behaviour, the sooner an intact anatomy is created, the better. ${ }^{7-10}$ As yet, however, there is no evidence that early surgery would lead to better speech development.

\section{Rationale}

The widespread uncertainty surrounding the timing of palatal closure is reflected in the diversity of protocols currently employed by the Scandcleft Research Group, a partnership of Scandinavian and UK cleft lip and palate centres. ${ }^{1-22}$ The Scandcleft Research Group identified this uncertainty as a priority research question for a future trial. Its aim was to determine whether, in infants with cleft palate, repair at either age 6 or 12 months (corrected for gestational age) would achieve better speech outcomes. The design of the trial was supported by a planning grant from the National Institute of Dental and Craniofacial Research (NIDCR), a substream of the US National Institutes of Health (NIH), who subsequently funded the proposed trial.

\section{Objectives}

The aim of this project is to determine whether, in infants with isolated cleft palate, it is better to perform primary surgery at age 6 or 12 months (corrected for gestational age). Gestational age will be assessed based on the date of the last menstrual period and the infant's date of birth (full term defined as day 1 of the 40th week of pregnancy), thus taking account of prematurity. This research will investigate the effect of the timing of surgery by assessing and comparing speech development outcomes measured across 12 months, 3 years and 5 years of age. In addition, secondary outcomes include growth, perioperative complications, dentofacial development, hearing level and middle ear function.

\section{METHODS AND ANALYSIS \\ Design}

Timing Of Primary Surgery for cleft palate (TOPS) is an international, multisite trial using a parallel arm design aiming to detect whether surgery at 6 months is superior to surgery at 12 months. Infants will be randomised to receive primary surgery for cleft palate using a standardised technique (the Sommerlad technique) ${ }^{23}$ at either age 6 or 12 months (corrected for gestational age). The study design of TOPS trial is illustrated in figure 1.

\section{Setting}

The trial will be conducted by the cleft palate teams based in centres across the UK, Scandinavia and Brazil. Criteria for selection of sites are based primarily on the ability to enrol a high volume of patients into the trial. A list of the TOPS trial sites is provided in table 1 . The cleft team at each centre generally includes cleft surgeon(s), nursing staff, cleft speech and language therapist(s) (SLT), clinical geneticist(s)/paediatrician(s), audiologist(s), orthodontist(s) and psychologist(s)/social worker(s).

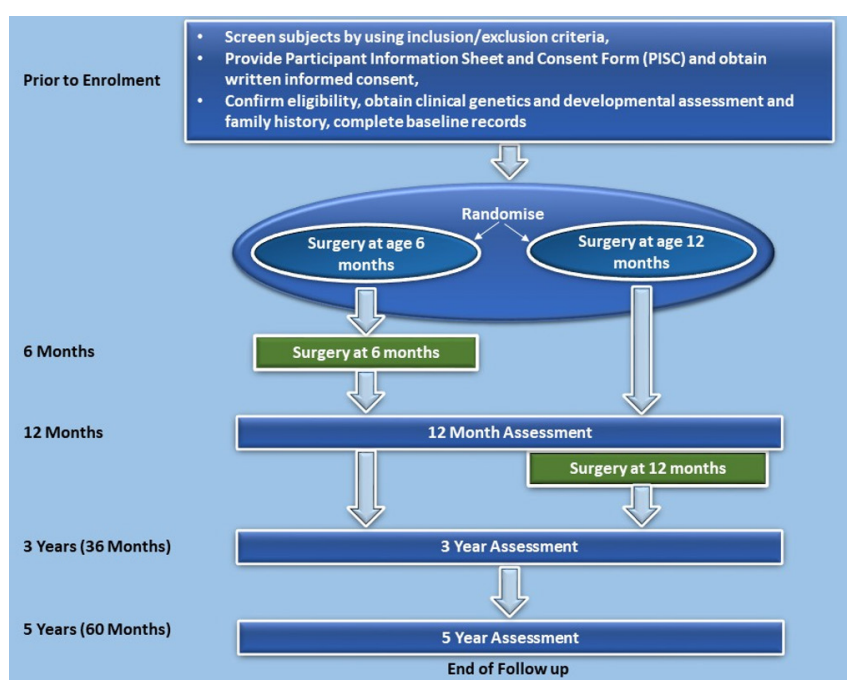

Figure 1 Flow diagram of Timing Of Primary Surgery for cleft palate study design.

\section{Eligibility criteria}

All infants referred to the participating specialised cleft lip and palate centres are eligible to enter the trial if they meet the following inclusion and none of the exclusion criteria:

a. Isolated cleft palate.

b. Medically fit for surgery at 6 months, corrected for gestational age.

c. Written informed proxy consent.

d. One parent/carer must be a native language speaker of the majority language in the country of residence.

Infants with any of the following will be excluded from the study:

a. Consent not obtained.

b. Infants with severe developmental delay (as measured on DENVER II) or syndromic cleft palate (except Van der Woude syndrome, which can be included if hearing is not affected) will be excluded.

c. Congenital sensorineural hearing loss or structural middle ear anomalies.

d. Sommerlad technique could not be performed due to variation in the anatomical presentation.

e. Infants presenting with submucous cleft palate (defined by the classical triad of signs, bifid uvula, bony defect of the hard palate, muscular diastasis, as described by Jensen et $a 2^{24}$ ).

f. Where the language spoken at home by at least one parent is not the majority language in the country of residence.

Since not all syndromic disorders will present prior to recruitment, all participants will undergo genetic testing to exclude chromosome abnormalities at the time of surgery. If a chromosome abnormality or another genetic syndrome is identified later in the study, the data for these participants will be handledas appropriate and in accordance with the presepecified Statistical Analysis Plan. The same will apply if the participant fails the DENVER II developmental test at 3-year follow-up. 


\begin{tabular}{ll}
\hline $\begin{array}{l}\text { Table 1 } \\
\text { clinical sites }\end{array}$ & Timing Of Primary Surgery for cleft palate trial \\
\hline Country & Sites \\
\hline Brazil & University of São Paulo (HRAC Bauru) \\
Denmark & $\begin{array}{l}\text { Copenhagen Cleft Palate Centre, Copenhagen } \\
\text { Århus Speech and Hearing Institute, Århus }\end{array}$ \\
Norway & Oslo University Hospital, Oslo \\
& Helse Bergen HF, Bergen \\
Sweden & Malmö University Hospital, Malmö \\
& Gothenburg University, Gothenburg \\
& Karolinska University Hospital, Stockholm \\
& University of Linköping, Linköping \\
& Umeå University, Umeå \\
& Uppsala University, Uppsala \\
& Royal Manchester Children's \\
& Hospital, Manchester \\
& Alder Hey Children's NHS Foundation \\
& Trust, Liverpool \\
& Royal Belfast Hospital for Sick Children, Belfast \\
& Birmingham Children's Hospital, Birmingham \\
& Royal Victoria Infirmary, Newcastle \\
& University Hospitals Bristol NHS Foundation \\
& Trust, Bristol \\
& Morriston Hospital, Abertawe Bro Morgannwg \\
& University Hospital Board, Swansea \\
& Leeds General Infirmary, Leeds \\
& Royal Hospital for Children, Glasgow \\
UK & Royal Hospital for Sick Children, Edinburgh \\
&
\end{tabular}

\section{Consent}

Informed consent will be sought from the infant's parent/guardian by a member of the local trial team. Families who decline to participate will receive surgery in line with the hospital's current practice together with the same level of care and support as families participating in the trial. Consent forms used in the TOPS trial in the UK are shown in online supplementary materials nos. 1, 2 and 3; these forms were adapted and translated for use in the other participating countries while maintaining key content. The final consent forms in Brazilian-Portuguese, Danish, Norwegian and Swedish were translated to English to check for accuracy and completeness. Participants can withdraw from the trial at any time without giving an explanation, and their child's care will not be affected.

To reduce potential burden to families, where possible, trial information will be collected at visits scheduled in line with routine visits made to the site as part of the infant's ongoing care.

\section{Randomisation}

Infants meeting the eligibility criteria will be randomised to 6-month or 12-month surgery, corrected for gestational age, in a ratio 1:1 using a minimisation routine incorporating a random element to reduce predictability. Allocations will be delivered via a password-protected web-based system.

Every effort will be made to arrange surgery within 1 week of the target date. However, surgery may take place up to 2 weeks before or 4 weeks after the target date. The estimated timing of surgery and the allowed time window for the surgery will be calculated by the online randomisation system and provided to the trial site at the time of randomisation.

\section{Interventions}

The Sommerlad surgical technique ${ }^{23}$ will be used in all participants at 6 or 12 months corrected for gestational as determined by randomisation. This technique will be standardised across all surgeons, including those who already use the technique, by receiving direct instruction from Mr Brian Sommerlad in the operating theatre. Written descriptions and a video of the surgical procedure will also be provided.

\section{Blinding}

The nature of the interventions prevents this trial from being blind to participants or their carers. However, speech outcome assessments, at ages 12 months, 3 and 5 years, will be rated blind to the randomly allocated group.

\section{Outcome measures}

The primary endpoint for the TOPS trial is defined as a dichotomous outcome of whether the child has been perceived by the SLTs to have insufficient velopharyngeal function at age 5 years or not. Adequate velopharyngeal function is a prerequisite for normal speech production. In children born with cleft palate, speech outcomes are often reported for velopharyngeal function and articulation.

In the presence of insufficient velopharyngeal function, speech will inevitably be affected by symptoms such as hypernasality and nasal air emission to different degrees. In children with isolated cleft palate, articulation disorders occur less frequently than in children with complete cleft lip and palate. Insufficient perceived velopharyngeal function was therefore chosen to be the primary outcome and articulation outcomes as secondary outcomes. Velopharyngeal insufficiency is measured by Velopharyngeal Composite Score (VPC) sum, which is an overall score on the scale $1-6^{25}$. Scores $\geq 4$ on this scale will be considered insufficient.

The secondary endpoints are summarised in box 1 and table 2 (Schedule of Assessments).

\section{Speech outcome assessments}

To ensure quality of speech data, all sites will receive identical high-quality recording equipment (video recorder JVG-GY-HM100 series, audio recorder H4n/H5 Handy 


\section{Box 1 Secondary endpoints}

1. Velopharyngeal function at age 5 years:

a. Velopharyngeal Composite Score summary (VPC sum).

b. Insufficient velopharyngeal function (VPC rate).

2. Velopharyngeal function at age 3 years:

a. Insufficient velopharyngeal function (VPC rate).

b. Velopharyngeal insufficiency symptoms.

3. Canonical babbling at age 12 months:
a. Canonical babbling present.
b. Canonical babbling ratio.
c. Consonant inventory.

4. Articulation at age 3 years:
a. Per cent consonants correct (PCC).
b. Per cent correct placement (PCP).
c. Per cent correct manner (PCM).
d. Non-oral consonant errors.
e. Oral consonant errors.

5. Articulation at age 5 years:
a. PCC.
b. PCP.
c. PCM.
d. Non-oral consonant errors.
e. Oral consonant errors.

6. Postoperative/long-term complications:
a. Dehiscence.
b. Infection.
c. Evidence of fistula.

7. Hearing level:

a. At 12 months:

i. Abnormal Transient Otoacoustic Emission (TEOAE).

ii. Abnormal sound-field audiometry.

b. At 3 and 5 years:

i. Abnormal pure tone audiometry in at least one ear.

ii. Abnormal pure tone audiometry in both ears.

iii. Severity of better ear (normal, mild, moderate, severe, profound).

8. Middle ear function:

a. Flat line Tympanogram in at least one ear (12 months, 3 years, 5 years).

b. Flat line Tympanogram in both ears (12 months, 3 years, 5 years).

9. Dentofacial development at age 5 years:

a. Soft tissue ANB (the angle between soft tissue nasion, A point and $B$ point on a profile photograph). ${ }^{32}$

b. Maxillary Arch Constriction score (using modified Huddart/ Bodenham scoring system). ${ }^{33}$

10. Growth at 12 months:
a. Nude weight.
b. Crown to heel length.
c. Occipitofrontal circumference.

recorder and microphone Rode NT4/NT5) to be used at each follow-up recording according to a detailed standard operating procedure. Before data collection starts at each follow-up age, all SLTs will participate in a 3-day calibration meeting. Afterwards, a series of video-audio practice recordings will be completed and quality checked. When sufficient recording quality has been reached, the site receives approval that they can start the trial recordings. To train the SLTs who are going to perform speech assessments, a specific procedure has been developed. This includes theoretical lectures on development of speech and language in children with cleft palate and methodological considerations on assessment/rating, and listener training with discussions and personal feedback. Before the assessments start, all SLTs need to pass a test with a specified level of intrarater and inter-rater reliability. They also have to pass a hearing test.

At 12 months of age, assessments will be done cross-linguistically. At age 3 and 5 years, SLT rating will be confined to records of children sharing the SLT's native language.

Vocalisations of 12-month-old children will be assessed with adjusted real-time listening, as described by Ramsdell et $a .^{26}$ The SLTs will listen to a 45 min video recording of a play session between the child and the carer, divided into two parts (22 min each). The SLT will register every syllable a child produces as canonical or not, in real time, using a software, TimeStamper, specifically developed for this study. ${ }^{27}$ At the end of each recording, the SLT indicates if the child babbled canonically or not and lists the syllables the child produced with control. In this way, the variables canonical babbling present, canonical babbling ratio and consonant inventory are obtained.

The methodology for the 5-year assessment of articulation and velopharyngeal function cross-linguistically was developed within the Scandcleft study ${ }^{122021}$ and will be extended to include Brazilian Portuguese. At the 5-year assessment, 36 target consonants from the TOPS single word test will be transcribed phonetically for assessment of articulation and Velopharyngeal Insufficiency (VPI) symptoms. Target words include similar target sounds in the same position and with similar phonetic context across languages. Further, repetition of sentences and continuous speech are collected, as well as nasalance scores (Nasometer), and parent-reported intelligibility estimates of how well their children's speech is understood by different listeners (Intelligibility in context scale $^{28}$ ). The 3 -year assessment will be based on 30 of the 36 words used in the 5 -year assessment, and target consonants will be transcribed phonetically for assessment of articulation. Error types will be classified automatically by a predefined script that will also allow calculation of percent consonant correct, per cent correct placement and percent correct manner. The VPC rate will be rated by SLTs from continuous speech both at age 3 and 5 years.

\section{Patient and public involvement}

Parents of children with cleft palate will be approached by their orthodontist/surgeonsprior to enrolment. Patients and their parents were not initially involved in the design of the study. However, a representative from the Cleft Lip \& Palate Association (the charity for Cleft Lip \& Palate in the UK) is a member of the Trial Steering Committee. Therefore, providing ongoing insight from a parent perspective with regard to the execution of this study and the dissemination of results. 
Table 2 Schedule of assessments

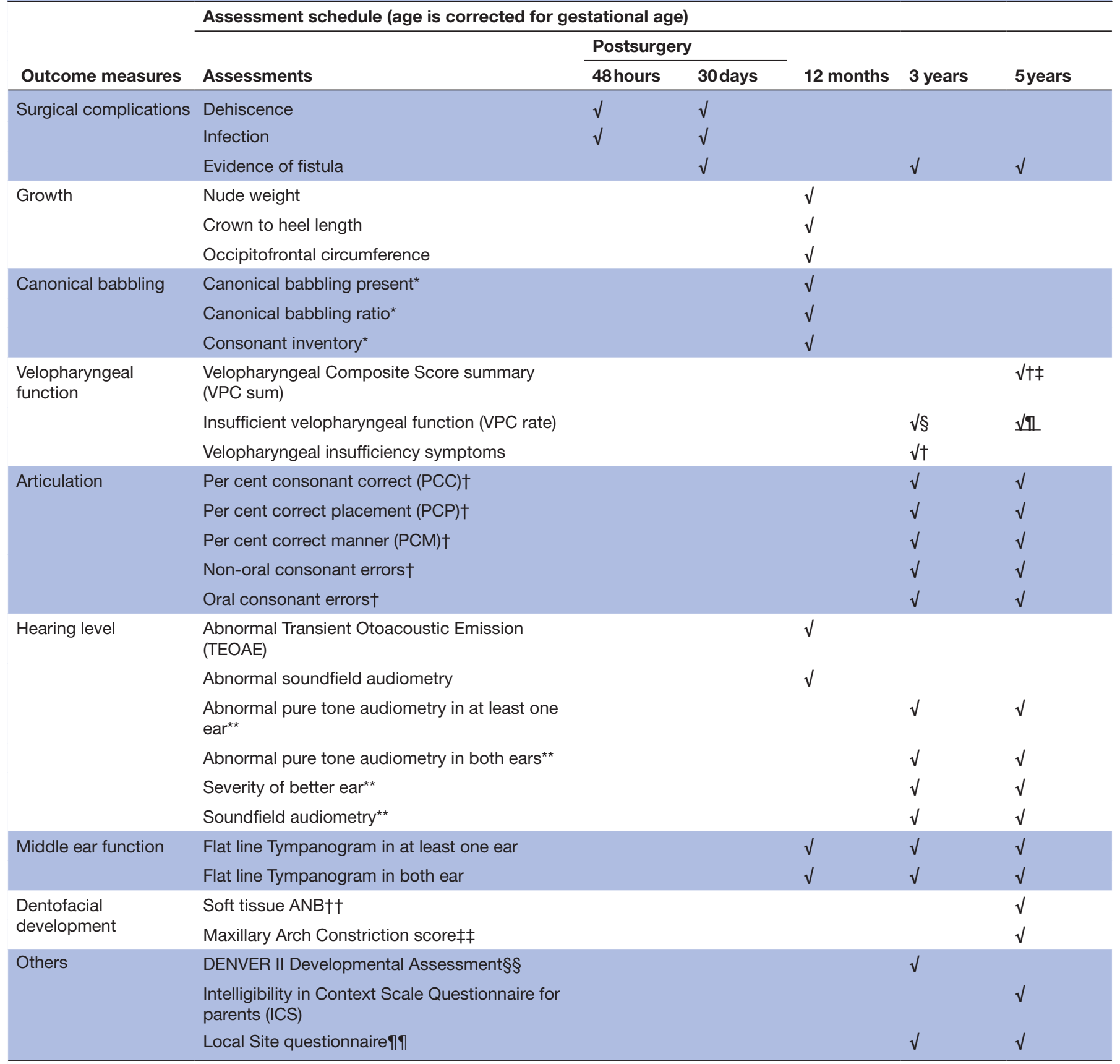

Sources of speech assessments:

* Video of play interaction.

†TOPS picture naming test.

$\ddagger$ Nine-word string.

$\S$ Spontaneous speech.

१:Spontaneous speech(retelling of bus story).

**If pure tone audiometry could not be performed, then soundfield audiometry will be performed.

††The angle between soft tissue nasion A point and B point on a profile photograph.

$\ddagger \ddagger$ Maxillary Arch Constriction score is determined using modified Huddart/Bodenham scoring system.

$\S \S D E N V E R$ II Developmental Assessment is carried out at the time of surgery.

ๆๆ Local site questionnaire sent to local speech and language therapists outside TOPS research team to collect data on direct and indirect therapy given to the child in the intervals between assessment visits.

\section{Data collection and management}

Trial data will be recorded on Case Report Forms (CRFs) and identifiable only by randomisation number. The data from completed CRFs will be entered onto the trial-specific MACRO database by the Data Manager or appropriately trained personnel at the Data Coordinating Centre. 


\section{Video and audio recording}

Once recorded, video and audio recordings will be saved onto encrypted USB drives. They will be posted to the Data Coordinating Centre where, on receipt, they will be logged and stored onto the trial-specific secure server. This server will be backed up once a day to ensure data are not lost once received. Recordings are quality checked by the Core Speech Group and/or the Trial Administrative Centre. A satisfactory recording is one that passes prespecified quality checks on lighting, length and sound. Quality checks will be performed regularly and feedback to site will be provided on their suitability for assessment.

\section{Maxillary arch impressions}

Maxillary arch impressions will be obtained at the time of surgery to provide a mould for plaster casts, which are sent to the TOPS Administrative Centre at the University of Manchester.

In addition, impressions of the maxillary and mandibular dental arches will be obtained at the 5-year follow-up appointment. Impressions are taken by a designated member of staff (usually the orthodontist) using appropriate impression material. The occlusion will be registered with a wax wafer in the position of maximal intercuspation. The study models made from the impressions will be stored at the TOPS Administrative Centre.

\section{Photographs}

Intraoral photographs will be taken at the time of surgery, and frontal and lateral photographs will be obtained at the 5-year visit. The photographs will be saved onto encrypted USBs, on receipt by the Data Coordinating Centre they will be logged and stored onto trial-specific secure hard drives.

\section{Statistical analysis and sample size considerations Proposed sample size}

Three hundred patients per arm will allow a reduction in insufficient velopharyngeal function at 5 years from $40 \%$ to $29 \%$ to be detected with $81 \%$ power using a chi-square test (two-sided significance test at 0.05 level). The estimate of $40 \%$ was obtained from a pilot trial of 50 patients of 5 years of age, collected during the planning period for this grant application. ${ }^{12}$ To allow an approximate drop out of $10 \%, 648$ participants will be recruited. However, to consider the potential impact of variability around the value of $40 \%, 300$ patients per group would provide $80 \%$ power to detect a reduction from $30 \%$ to $20 \%$ and $76 \%$ power to detect a reduction from $20 \%$ to $12 \%$.

The trial enrolment, allocation, follow-up and analysis will be reported using the 'Consolidated Standard of Reporting Trials' ('CONSORT') ${ }^{29}$ and the International Conference on Harmonisation E9 guidelines. ${ }^{30} \mathrm{~A}$ full and detailed statistical analysis plan ${ }^{31}$ will be developed prior to the final analysis of the trial. The main features of the statistical analysis plan are included here.

The primary analysis will be by intention-to-treat principle, as far as is practically possible using a $5 \%$ significance level throughout. Rather than adjust for multiplicity of secondary outcomes, relevant results from other studies already reported in the literature will be taken into account in the interpretation. The approach to formal analyses will be dependent on outcome type as follows:

- Dichotomous outcome will be compared between the two groups using a chi-squared test and the effect estimate will be reported in terms of the relative risk and $95 \%$ confidence interval.

- Short ordinal outcomes will be compared using a chi-squared test for trend.

- Continuous and long ordinal outcomes will be compared between the two groups using a two group t-test. The difference in means will be presented with a $95 \%$ CI.

Baseline and operative characteristics and safety data will be presented using descriptive statistics only.

If the percentage of major protocol deviations exceeds $10 \%$ and the trial management group consider this analysis appropriate, a per-protocol analysis in which prespecified major protocol deviations indicate exclusion of a participant from the analysis set will be conducted.

\section{Trial oversight and monitoring}

The Trial Management Group (TMG), Trial Steering Committee (TSC) and Data Safety and Monitoring Board (DSMB) will provide ongoing oversight and will monitor accruing trial data. The roles, responsibilities and composition of each of these committees are provided in online supplementary material no. 4. A risk assessment has been conducted and used to inform a trial-specific monitoring plan agreed by the independent oversight committees.

\section{Trial status and timeline}

The overall programme commenced on 13 July 2010. Applications for ethics approval were submitted on 10 November 2009. Recruitment to the trial commenced on 13 July 2010. Participants will be followed up until 30 July 2020.

This trial completed recruitment on 21 July 2015, and the last patient is due to attend their last visit until 30 of July 2020.

\section{ETHICS}

Ethical approval has been sought in each participating country according to country-specific procedures. The protocol has gained favourable opinion from the Multicentre Research Ethics Committee in the UK and from relevant ethics committees for each participating centre. TOPS Protocol Version 1.1 (of 2 November 2009) was approved by UK ethics on 8 January 2010, the Protocol Version 4.0 (of 26 August 2015) was approved by UK ethics on 1 October 2015 and the Protocol Version 5.0 (of 22 August 2018) was approved by UK ethics on 18 November 2018. A summary of substantial protocol amendments and relevant ethics committees is provided in online supplementary material no. 5 . 


\section{Dissemination}

Following completion of the study, the principal investigator is expected to publish the results of this research in a peer-reviewed scientific journal. According to the NIH Public Access Policy, all journal articles arising from this NIH-funded trial will be submitted to the digital archive PubMed Central. Trial investigators have the right and responsibility to communicate their findings to the scientific community and to the public. Findings of the trial will also be presented at National and International meetings of relevant professional bodies and research groups. Reports will also be posted on the WHO website (www. who.org) craniofacial section. Access to clinical data sets within speech, genetic, surgical and other fields will be available to others following the acceptance for publication of the main findings from the final dataset. Requests to access data will be subject to participant confidentiality concerns and to contemporary NIH guidance on datasharing plans.

\section{Author affiliations}

${ }^{1}$ School of Medical Sciences, Division of Dentistry, The University of Manchester, Manchester, Greater Manchester, UK

${ }^{2}$ Functional Area Speech and Language Pathology, Division of Speech and Language Pathology, Karolinska Institute, Stockholm, Sweden

${ }^{3}$ Institute of Neuroscience and Physiology, Speech and Language Pathology Unit, Sahlgrenska Academy, University of Gothenburg, Gothenburg, Sweden

${ }^{4}$ Department of Nordic Studies and Linguistics, University of Copenhagen, Copenhagen, Denmark

${ }^{5}$ Division of Evolution and Genomic Sciences and Manchester Centre for Genomic Medicine, Manchester University NHS Foundation Trust, St Mary's Hospital,

Manchester, Greater Manchester, UK

${ }^{6}$ Hospital de Reabilitação de Anomalias Craniofaciais Universidade de São Paulo, 5Facu Faculdade de Odontologia de Bauru, Bauru-SP, Brazil

${ }^{7}$ Manchester Centre for Audiology and Deafness, School of Health Sciences, The University of Manchester, Manchester, Greater Manchester, UK

${ }^{8}$ Clinical Trials Research Centre, University of Liverpool, Liverpool, UK

Acknowledgements The authors acknowledge our partners in Cleft Care centres in UK, Scandinavia and Brazil for participating in data collection and the SLTs for their contributions in calibration and rating of speech assessments during various stages of the TOPS trial. KJM is supported by the NIHR Manchester Biomedical Research Centre.

Contributors WS, the chief investigator, and GS conceived the trial as an extension of the Scandcleft Trial and developed the first version of the protocol with AL, EW, CP, PW and CG. JC-S developed the genetic aspects of the protocol and IKT provided logistic advice for extension of the study to Brazil. NH and DW contributed to coordination and implementation of the study and revised and finalised the study protocol and KJM and EJC participated in writing the protocol. All authors reviewed and approved this manuscript.

Funding This publication was made possible by Grants Number R21DE15128, U01DE018664 and U01DE018837 from the National Institute of Dentaland Craniofacial Research (NIDCR). Its contents are solely the responsibility of the authors and do not necessarily represent the official views of the NIDCR.

Competing interests None declared.

\section{Patient consent for publication Obtained}

Ethics approval Ethical approval has been sought in each participating country according to country specific procedures. The protocol has gained favourable opinion from the Multicentre Research Ethics Committees in the UK, Denmark, Sweden, Norway and Brazil. The trial will abide by the principles of the World Medical Association Declaration of Helsinki (1964) and the Tokyo (1975), Venice (1983), Hong Kong (1989) and South Africa (1996), the Office of Human Research Protections (OHRP) Common Rule, 45 CFR 46 and General Data Protection Regulations (GDPR), accompanied by UK Data Protection Act (2018).
Provenance and peer review Not commissioned; externally peer reviewed.

Open access This is an open access article distributed in accordance with the Creative Commons Attribution Non Commercial (CC BY-NC 4.0) license, which permits others to distribute, remix, adapt, build upon this work non-commercially, and license their derivative works on different terms, provided the original work is properly cited, appropriate credit is given, any changes made indicated, and the use is non-commercial. See: http://creativecommons.org/licenses/by-nc/4.0/.

\section{REFERENCES}

1. Tanaka SA, Mahabir RC, Jupiter DC, et al. Updating the epidemiology of isolated cleft palate. Plast Reconstr Surg 2013;131:650e-2.

2. Peterson-Falzone S. Optimal age for palatoplasty to faclitate normal speech development: What is the evidence? In: Berkowitz S, ed. Cleft Lip and Palate. Berlin: Springer, 2006:691-700.

3. Hellquist R, Pontén B, Skoog T. The influence of cleft length and palatoplasty on the dental arch and the deciduous occlusion in cases of clefts of the secondary palate. Scand J Plast Reconstr Surg 1978;12:45-54.

4. Friede $\mathrm{H}$, Persson EC, Lilja J, et al. Maxillary dental arch and occlusion in patients with repaired clefts of the secondary palate. Influence of push back palatal surgery. Scand J Plast Reconstr Surg Hand Surg 1993;27:297-305.

5. Nyström M, Ranta R. Effect of timing and method of closure of isolated cleft palate on development of dental arches from 3 to 6 years of age. Eur J Orthod 1994;16:377-83.

6. Friede H, Enocson L, Möller M, et al. Maxillary dental arch and occlusion in repaired clefts of the secondary palate: influence of surgical closure with minimal denudation of bone. Scand J Plast Reconstr Surg Hand Surg 2000;34:213-8.

7. Dorf DS, Curtin JW. Early cleft palate repair and speech outcome. Plast Reconstr Surg 1982;70:74-9.

8. Chapman KL, Hardin-Jones MA, Goldstein JA, et al. Timing of palatal surgery and speech outcome. Cleft Palate Craniofac $J$ 2008;45:297-308.

9. Chapman KL, Willadsen E. The Development of Speech in Children with Cleft Palate. Cleft Palate Speech: Assessment and Intervention. 2011:23-40.

10. Jones DL. Timing of palatoplasty and speech. In: Comprehensive Cleft Care: Taylor \& Francis Group, LLC, 2016:521-6.

11. Semb G, Enemark H, Friede $H$, et al. A Scandcleft randomised trials of primary surgery for unilateral cleft lip and palate: 1. Planning and management. J Plast Surg Hand Surg 2017;51:2-13.

12. Lohmander A, Willadsen E, Persson C, et al. Methodology for speech assessment in the Scandcleft project--an international randomized clinical trial on palatal surgery: experiences from a pilot study. Cleft Palate Craniofac J 2009;46:347-62.

13. Bannister $P$, Lindberg $N$, Jeppesen $\mathrm{K}$, et al. Scandcleft randomised trials of primary surgery for unilateral cleft lip and palate: 3 . Descriptive study of postoperative nursing care following first stage cleft closure. J Plast Surg Hand Surg 2017;51:21-6.

14. Feragen KB, Rumsey N, Heliövaara A, et al. Scandcleft randomised trials of primary surgery for unilateral cleft lip and Palate: 9. Parental report of social and emotional experiences related to their 5-year-old child's cleft diagnosis. J Plast Surg Hand Surg 2017;51:73-80.

15. Feragen KB, Semb G, Heliövaara A, et al. Scandcleft randomised trials of primary surgery for unilateral cleft lip and palate: 10. Parental perceptions of appearance and treatment outcomes in their 5-yearold child. J Plast Surg Hand Surg 2017;51:81-7.

16. Heliövaara A, Küseler A, Skaare $P$, et al. Scandcleft randomised trials of primary surgery for unilateral cleft lip and palate: 6 . Dental arch relationships in 5 year-olds. J Plast Surg Hand Surg 2017;51:52-7.

17. Mølsted K, Humerinta K, Küseler A, et al. Scandcleft randomised trials of primary surgery for unilateral cleft lip and palate: 8 . Assessing naso-labial appearance in 5-year-olds - a preliminary study. J Plast Surg Hand Surg 2017;51:64-72.

18. Rautio J, Andersen M, Bolund S, et al. Scandcleft randomised trials of primary surgery for unilateral cleft lip and palate: 2 . Surgical results. J Plast Surg Hand Surg 2017;51:14-20.

19. Shaw W, Semb G. The Scandcleft randomised trials of primary surgery for unilateral cleft lip and palate: 11. What next? J Plast Surg Hand Surg 2017;51:88-93.

20. Willadsen E, Lohmander A, Persson C, et al. Scandcleft randomised trials of primary surgery for unilateral cleft lip and palate: 5 . Speech outcomes in 5-year-olds - consonant proficiency and errors. J Plast Surg Hand Surg 2017;51:38-51.

21. Lohmander A, Persson C, Willadsen E, et al. Scandcleft randomised trials of primary surgery for unilateral cleft lip and palate: 4 . Speech 
outcomes in 5-year-olds - velopharyngeal competency and hypernasality. J Plast Surg Hand Surg 2017;51:27-37.

22. Karsten A, Marcusson A, Hurmerinta K, et al. Scandcleft randomised trials of primary surgery for unilateral cleft lip and palate: 7 . Occlusion in 5 year-olds according to the Huddart and Bodenham index. J Plast Surg Hand Surg 2017;51:58-63.

23. Sommerlad BC. The use of the operating microscope for cleft palate repair and pharyngoplasty. Plast Reconstr Surg 2003;112:1540-1.

24. Jensen BL, Kreiborg S, Dahl E, et al. Cleft lip and palate in Denmark, 1976-1981: epidemiology, variability, and early somatic development. Cleft Palate $J$ 1988;25:258-327.

25. Lohmander A, Hagberg E, Persson C, et al. Validity of auditory perceptual assessment of velopharyngeal function and dysfunction the VPC-Sum and the VPC-Rate. Clin Linguist Phon 2017;31:589-97.

26. Ramsdell $\mathrm{HL}$, Oller DK, Buder EH, et al. Identification of prelinguistic phonological categories. J Speech Lang Hear Res 2012;55:1626-39.

27. Willadsen E, Persson C, Appelbe D. A software program to assist coding of prelinguistic vocalizations in real time. Clin Linguist Phon 2018;32:972-8.
28. McLeod S, Harrison LJ, McCormack J. The intelligibility in Context Scale: validity and reliability of a subjective rating measure. $J$ Speech Lang Hear Res 2012;55:648-56.

29. Schulz KF, Altman DG, Moher D. CONSORT Group. CONSORT 2010 statement: updated guidelines for reporting parallel group randomized trials. Ann Intern Med 2010;152:726-58.

30. ICH Harmonised Tripartite Guidline. Statistical principles for clinical trials. International Conference on Harmonisation IE9 Expert Working Group. Stat Med 1999;18:1905-42.

31. Gamble C, Krishan A, Stocken D, et al. Guidelines for the Content of Statistical Analysis Plans in Clinical Trials. JAMA 2017;318:2337-43.

32. Bearn DR, Sandy JR, Shaw WC. Photogrammetric assessment of the soft tissue profile in unilateral cleft lip and palate. Cleft Palate Craniofac J 2002;39:597-603.

33. Martin CB, MaX, McIntyre GT, et al. The validity and reliability of an automated method of scoring dental arch relationships in unilateral cleft lip and palate using the modified Huddart-Bodenham scoring system. Eur J Orthod 2016;38:353-8. 